\title{
OBSERVATIONS SUR L'EMPLOI ET L'EXPLOITATION DE LA METHODE DE LURY DANS L'INVENTAIRE PISCICOLE D'UNE RIVIERE DES LANDES
}

\author{
M. LAURENT (*), S. TRABUCHET, F. GROSS ("*) \\ (*) Station d'Hydrobiologie \\ I.N.R.A., B.P. 79 \\ 64200 - BIARRITZ \\ (**) Parc naturel régional des Landes de Gascogne \\ Préfecture, 40011 - MONT-DE-MARSAN
}

\section{RES U M E}

Le peuplement piscicole de la Petite Leyre a èté examiné. On a trouvé cinq espèces de poissons : Phoxinus phoxinus L. (vairon) - Nemacheilus barbatulus L. (loche) - Esox lucius L. (brochet) - Salmo irideus G. (truite arc-en-ciel) Anguilla anguilla $L$. (anguille). Pour les anguilles on a pu évaluer le peuplement (57 kg/ha) à l'aide de la méthode "de LURY * (4 pêches successives).

Dans le but de connaitre la composition qualitative et quantitative du peuplement piscicole de la Petite Leyre, un inventaire par la méthode * de LURY * a été réalisé. II a permis d'évaluer le peuplement d'anguilles, en effectuant quatre pêches successives; on a pu, à cette occasion, montrer l'intérêt d'effectuer le plus de pêches possible pour améliorer la précision des inventaires.

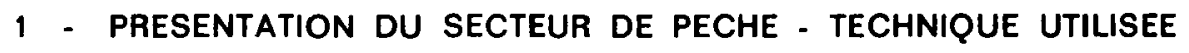

Le secteur de pèche a les caractéristiques suivantes:

- Rivière : Petite Leyre

- Longueur et situation : $150 \mathrm{~m}$ en amont du pont situé sur la route de BELHADE à MONTAUZEY (Carte Michelin no 78) 
- Largeur moyenne et surface du secteur : $9 \mathrm{~m}-0,135$ ha

- Etat de la rivière - profondeur moyenne : fond sableux uniforme sur toute la surface, ce qui entraine la pauvreté de la faune benthique. La rivière coule sous un couvert végétal important qui empêche le réchauffement de l'eau. La profondeur moyenne est de $0,60 \mathrm{~m}$.

- Date de l'opération : 9 et 10 Juillet 1974

- Température de l'eau : 16 ○ $\mathrm{C}$.

La faible minéralisation des eaux de cette rivière (résistivité : $9500 \Omega / \mathrm{cm}$ ) en fait, comme toutes les rivières de ce type, un biotope peu productif.

Techniques : L'appareil de pêche électrique est le Martin-Pêcheur, décrit par GOSSET, LAMARQUE et CHARLON (1971); l'inventaire est un inventaire de type de LURY. Tous les poissons ont été mesurés après anesthésie dans la cuve galvanonarcotique (GOSSET, 1974). Les pesees n'ayant pu être faites, on a utilisé pour les anguilles une relation longueur-poids établie pour la rivière Le Magescq, rivière du même type que la Petite Leyre, par CHARLON, LAMARQUE et LAURENT (à paraitre). Les estimations du peuplement et les limites de confiance n'ont pu être calculées que pour les anguilles; pour les autres espèces seules des indications sont données. Quelques contenus stomacaux sont également présentés à titre indicatif.

\section{II - PEUPLEMENT PISCICOLE} trouvées :

Outre les anguilles, que nous verrons plus loin, quatre espèces ont été

- Vairons (Phoxinus phoxinus L.) : en grande quantité - sur 51 individus examinés la taille moyenne est de $4 \mathrm{~cm}$, un seul individu dépassant $6 \mathrm{~cm}$.

- Loches (Nemacheilus barbatulus L.) : en petite quantité - 8 individus seulement ont èté capturès (taille moyenne $2 \mathrm{~cm}$ ).

- Brochets (Esox lucius L.) : 2 brochetons ont été capturés (tailles : $23 \mathrm{~cm}$ et $37 \mathrm{~cm})$.

- Truites arc-en-ciel (Salmo irideus G.) : il s'agit là d'un peuplement très particulier puisque les 5 individus capturés le 10 juillet provenaient d'un déversement dit * surdensitaire ^ effectué environ $1 \mathrm{~h}$ avant la pêche. En réalité, un tel déversement ne peut être considéré comme surdensitaire puisque la densité avaint déversement est nulle. Il serait plus exact de parler d'introduction temporaire, les truites arc-en-ciel non capturées par les pêcheurs étant destinées à être rapidement éliminées.

\section{III - ETUDE DU PEUPLEMENT D'ANGUILLES}

La répartition des anguilles capturées au cours des différentes pêches en classes de taille de $4 \mathrm{~cm}$ est présentée dans le Tableau 1.

Pour effectuer le calcul on supposera, en première approximation, que l'efficacité ne dépend pas de la taille et on regroupera tous les individus. La détermination du peuplement et de ses limites de confiance étant présentée de façon complète par LAURENT et LAMARQUE (1974), seul le principe en est rappelé : on calcule la droite de régression obtenue en considérant le nombre de poissons capturés à la pêche d'un numéro donné, comme une fonction du 


\begin{tabular}{|c|c|c|c|c|c|c|c|}
\hline \multirow{2}{*}{ Classe de taille } & \multicolumn{7}{|c|}{ Numéro des pêches } \\
\hline & fere pêche & $2^{e}$ & pêche & 3e pêche & $4 e$ & pêche & TOTAL \\
\hline $8-11,9$ & 1 & & 0 & 0 & & 0 & 1 \\
\hline $12-15,9$ & 1 & & 4 & 1 & & 0 & 6 \\
\hline $16-19,9$ & 4 & & 2 & 0 & & 1 & 7 \\
\hline $20-23,9$ & 5 & & 5 & 3 & & 2 & 15 \\
\hline $24-27,9$ & 5 & & 4 & 3 & & 1 & 13 \\
\hline $28-31,9$ & 10 & & 6 & 1 & & 0 & 17 \\
\hline $32-35,9$ & 4 & & 2 & 1 & & 2 & 9 \\
\hline $36-39,9$ & 7 & & 5 & 4 & & 0 & 16 \\
\hline $40-43,9$ & 2 & & 2 & 0 & & 1 & 5 \\
\hline $44-47,9$ & 4 & & 2 & 2 & & 0 & 8 \\
\hline TOTAL & 43 & & 32 & 15 & & 7 & 97 \\
\hline
\end{tabular}

Tableau 1. - Nombre d'anguilles capturées, réparties en classes de taille de $4 \mathrm{~cm}$, au cours des 4 pêches.

nombre cumulé de poissons capturés jusqu'à la pêche précédente. L'abscisse du point d'intersection de cette droite et de l'axe des $x$ est le nombre le plus probable de poissons. Une estimation des limites de confiance est obtenue par intersection avec l'axe des abscisses de deux droites, qui peuvent être considérées comme les limites à 95 p. 100 de la droite de régression. Si l'effectif cumulé des captures est supérieure à la limite inférieure, on prend évidemment ce dernier comme limite inférieure (voir fig. 1).

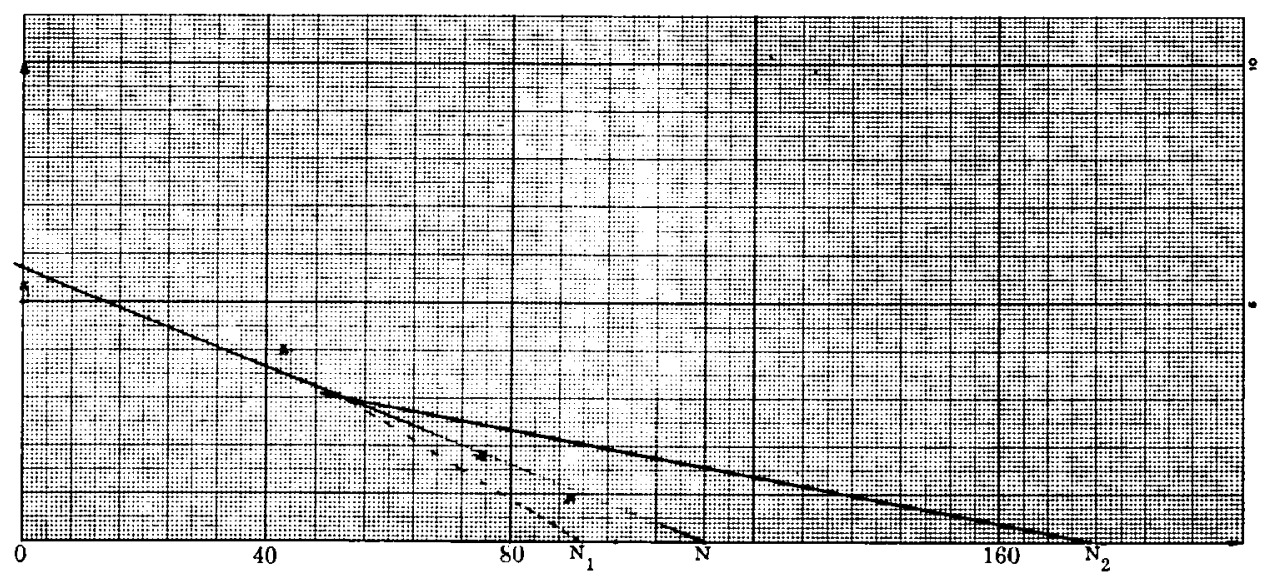

\section{LEGENDE DE LA FIGURE I}

Droite de régression permettant le calcul du peuplement le plus probable $N$ et de la limite supérieure $N_{2}-$ En pointillé droite donnant la limite inférieure (inférieure à la somme des captures $N_{1}$ ).

On obtient les résultats suivants en regroupant toutes les anguilles :

Nombre le plus probable: $N=112$

Limite inférieure $\quad: N_{1}=97$

Limite supérieure $\quad: N_{2}=175$

Pour l'évaluation du poids $p$ on utilise la relation longueur-poids, déterminée par CHARLON, LEMARQUE et LAURENT (à paraitre) sur les anguilles du Magescq (ruisseau alimentant l'étang de Soustons, du même type que la 
Petite Leyre) $\log p=-7,30+3,31 \log L$ ou en logarithmes décimaux $\log p=3,17+3,31 \log L$. On évalue ainsi le poids moyen pour chaque catégorie de longueur, on répartit les nombres $N, N_{1}$ et $N_{2}$ dans chaque catégorie de longueur proportionnellement à l'importance de ces derniers dans l'échantillon total (équivalent à $N_{1}$ ).

Le résultat final de l'estimation en poids est :

$$
P_{1}=6.7 \mathrm{~kg}<P=7,7 \mathrm{~kg}<P_{2}=12,1 \mathrm{~kg}
$$

Limite inférieure $P_{1}=6,7 \mathrm{~kg}$

Limite supérieure $P_{2}=12,1 \mathrm{~kg}$

et par ha (surface du secteur: $0,135 \mathrm{ha}$ )

$F_{1 \text { ha }}=49,6 \mathrm{~kg}<\mathrm{P}_{\mathrm{ha}}=57 \mathrm{~kg}<\mathrm{P}_{2 \mathrm{ha}}=89,6 \mathrm{~kg}$.

\section{Intérèt d'effectuer quatre pêches}

Le fait d'avoir fait quatre pêches a permis de calculer le peuplement le plus probable et les limites de confiance.

a) Si on s'ètait contenté des 2 premières pèches, la condition de SEBER et LE CREN :

$$
\frac{C^{2}}{C_{1}{ }_{2}} \frac{\left(C_{1}-C_{2}\right)^{2}}{\left(C_{1}+C_{2}\right)}>16
$$

ne serait pas satisfaite, avec $C_{1}=43$ et $C_{2}=32$, on pourrait donc seulement dire $N \geqslant 75$.

b) $\mathrm{Si}$ on s'était contenté des 3 premières pêches, on trouverait pour le coefficient de corrélation de la droite de régression la valeur $r=0,978<0.997$, valeur limite; on pourrait seulement conclure que $N \geqslant 90$.

c) $\mathrm{Si}_{1}$ comme cela est parfois pratiqué (de MOFFARTS, 1974), et ce qui est possible statistiquement, on avait considéré l'ensemble des 2 premières pêches comme une première pêche (de LURY), et l'ensemble des 2 dernières comme une deuxième pèche, la condition de SEBER et LE CREN :

$$
\frac{C^{1}}{C^{2}} \frac{\left(C_{1}-C_{2}\right)^{2}}{\left(C_{1}+C_{2}\right)}>16 \text { avec } C_{1}=75 \text { et } C_{2}=22
$$

serait satisfaite, et on pourrait estimer :

$$
\begin{aligned}
& \qquad N=\frac{C^{2}{ }_{1}}{C_{1}-C_{2}}=\frac{(75)^{2}}{53}=106 \\
& \text { et l'écart-type } J=\frac{C_{1} C_{2} \sqrt{C_{1}+C_{2}}}{\left(C_{1}-C_{2}\right)^{2}}=\frac{75 \times 22 \sqrt{97}}{(53)^{2}}=6 \\
& \text { et les limites de confiance : } 97 \leqslant N \leqslant 118,
\end{aligned}
$$

97 ètant la limite inférieure obtenue par addition des captures. Si on compare ces derniers résultats avec ceux obtenus précédemment, on constate que le peuplement le plus probable est sous-estimé.

\section{IV - CONTENUS STOMACAUX}

Les organismes suivants ont été trouvés dans les estomacs de 12 anguilles sacrifiè : 
a) Larves d'insectes : Odonates (aeschne), Trichopteres, Coléoptères, Phryganes, Diptères

b) Crustacés : Gammares

c) Insectes adultes : Coléopteres (gyrins), Orthoptères

d) Poissons : Vairons

\section{$V$ - CONCLUSION}

Le peuplement piscicole de la Petite Leyre est relativement pauvre qualitativement (cinq espèces de poissons dont l'une (truite arc-en-ciel) ne devait absolument pas sa présence aux conditions naturelles). A l'occasion de l'étude des anguilles, on a pu mettre en évidence la nécessité d'effectuer 4 pêches successives pour pouvoir évaluer le peuplement et ses limites de confiance. dans le cas d'un peuplement faible et d'une efficacité relativement faible (pente de la droite de régression 0,41 en moyenne).

L'examen des contenus stomacaux montre que les larves d'insectes sont consommées et, en particulier, les larves d'odonates souvent dédaignées par les autres espèces à cause de la dureté de leurs téguments.

\section{REMER CIEMENTS}

Nous remercions les membres de l'équipe d'animation du Parc de la Leyre pour l'aide qu'ils nous ont apportée.

\section{B I B L I O G R A P H I E}

CHARLON N., LAMARQUE P., LAURENT M., à paraitre. Etude de la population d'anguilles de l'Etang de Soustons (Landes).

GOSSET C., 1974. Cuve galvanonarcotique pour la mensuration des poissons. Bull. Fr. Piscic., 255.

GOSSET C., LAMARQUE P., CHARLON N., 1971. Un nouvel appareil de pêche électrique portable : le "Martin-Fêcheur". Bull. Fr. Piscic., 242, 33-46.

LAURENT M., LAMARQUE P., 1974. Utilisation de la methode des captures successives (de LURY) pour l'évaluation des peuplements piscicoles. Ann. Hydrobiol., 5, 2 (sous presse).

MOFFARTS (de) E., 1974. Densitè et biomasse des populations de poissons de la Haute-Sambre (MONCEAU-LOBBES). Congr. Ass. Fr. Limnol., Les Eyzies, mai 1974,3 p., ronéotypé. 